\title{
On the fracture models determined by the continuum-strong discontinuity approach
}

\author{
A.E. HUESPE ${ }^{1, *}$, J. OLIVER ${ }^{1}$, M.D.G. PULIDO ${ }^{1}$, S. BLANCO ${ }^{1}$ and \\ D. LINERO ${ }^{1,2}$ \\ ${ }^{1}$ E.T.S. d' Enginyers de Camins, Canals $i$ Ports, Technical University of Catalonia (UPC), Campus \\ Nord UPC, Módul C1, c/Jordi Girona 1-3, 08034 Barcelona, Spain \\ ${ }^{2}$ National University of Colombia, Engineering School, Bogotá, Colombia \\ (*Author for correspondence. E-mail: huespe@cimne.upc.es)
}

Received 23 August 2005; accepted in revised form 6 September 2005

\begin{abstract}
The paper focuses on the Continuum Strong Discontinuity Approach (CSDA) to fracture mechanics, and the traction-separation cohesive laws induced from continuum dissipative models as their projections onto the failure interface. They are compared with the cohesive laws commonly used for the fracture simulation in quasi-brittle materials, typically concrete. Emphasis is placed in the analysis of the mechanical stress-strain states induced by the CSDA into the fracture process zone: first when the damage mechanism is initiated and, after, when the cohesive model determines the crack response. The influence of the material parameters, particularly the fracture energy and the initial continuum softening modulus, in the obtained phenomenological responses is also analyzed. Representative numerical solutions of fracture problems are finally presented.
\end{abstract}

Key words: Continuum strong discontinuity approach, cohesive models, computational fracture mechanics.

\section{Introduction}

The performance of classical cohesive traction-separation laws in modeling the appearance and progression of fractures is already a well known issue, and their benefits and drawbacks have been established in many works (Hillerborg 1985; Bazant, 2002; Bazant and Planas, 1998; Planas et al., 2003; Bazant and Li 1997). This paper focuses on a more recent modeling environment: the Continuum Strong Discontinuity Approach (CSDA) to fracture mechanics (Simo et al., 1993; Oliver, 1996a, 1996b; Oliver et al., 1999, 2003, 2004; Oliver and Huespe, 2004) and the cohesive models that are projected by that approach, onto the fracture interfaces, as degenerations of continuum (stress vs. strain) dissipative constitutive models. Therefore, in this sense there is a clear connection of the CSDA with the cohesive fracture mechanics. However, in the CSDA those traction separation laws are never explicitly implemented and the analysis is held in a continuum format in all the stages of the fracture process modeling. This work is an attempt of displaying some benefits, regarding the numerical simulation of fracture that can be extracted from this fact, namely:

- The CSDA may reproduce with higher resolution the dissipative phenomena occurring at the fracture process zone. It is not necessary to introduce an 


\section{A.E. Huespe et al.}

explicit distinction of the continuum behavior in the bulk and the discontinuous behavior in the fracture to model the transition from continuity to discontinuity.

- The so called time continuity (Papoulia et al., 2003) of the traction separation law is automatically achieved in the CSDA with no necessity of encoding the traction separation law.

- Some numerical problems (i.e., oscillations of the traction profiles along the crack paths) typical of cohesive models equipped with large initial dummy stiffness do not appear in the CSDA implemented with embedded strong discontinuity finite elements.

- The continuum dissipative mechanisms naturally modeled in the CSDA translate into smoother stress fields in the neighborhood of the crack path; unphysical results like the stress mismatch in cohesive models and the inconsistent traction profiles can be completely removed.

In summary, the CSDA could be regarded as a more comprehensive procedure, to model cohesive cracks, than simple traction separation laws introduced in an ad hoc manner. As it will be shown by means of some representative simulations, the pay off for the slightly more complex implementation involved in the CSDA, is an increasingly robustness and accuracy of the simulation of the fracture mechanics phenomena.

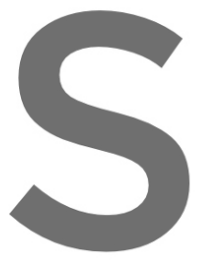

\section{The continumun-strong}

Although the CSDA has 1993; Oliver, 1996a, 1996 ; in this section it main
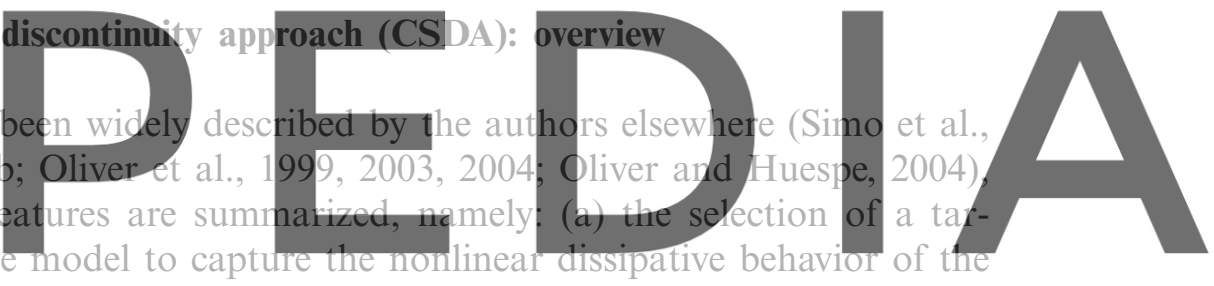

material and (b) the use of the so called strong discontinuity kinematics entailing a

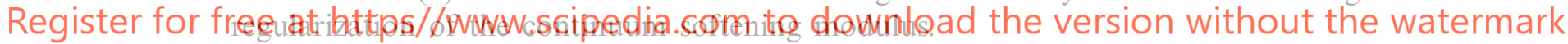

\subsection{TARget Constitutive MOdel: COntinuUm Damage}

There is no intrinsic restriction about the target continuum constitutive model, other than its adequacy to reproduce the phenomenological observed material behavior. In this sense, plasticity models for metallic or soil materials have been successfully used. Frictional materials failing in tension, like concrete, can be quite appropriately modeled by using continuum damage models and this motivates this particular choice. Let us, then, define an isotropic continuum damage constitutive model (Oliver, 2000), in terms of the following function of density of free energy:

$$
\varphi(\boldsymbol{\varepsilon}, r)=\frac{1}{2}\left(\frac{q(r)}{r} \boldsymbol{\varepsilon}: \mathbf{C}: \boldsymbol{\varepsilon}\right) ; \quad \mathbf{C}=\lambda(\mathbf{1} \otimes \mathbf{1})+2 \mu \mathbf{I}
$$

where $q \geqslant 0$ and $r \geqslant 0$ stand for the stress-like and strain-like internal variables, $\varepsilon$ is the strain tensor, $\mathbf{C}$ is the standard elastic constitutive tensor, depending on the Lamé's parameters $\lambda$ and $\mu$ and $\mathbf{1}$ and $\mathbf{I}$ being the unit second and fourth order tensors. The free energy function is then a potential for the stress field $\sigma$ defined as: 


$$
\boldsymbol{\sigma}=\Sigma(\boldsymbol{\varepsilon}, r)=\frac{\partial \varphi(\boldsymbol{\varepsilon}, r)}{\partial \boldsymbol{\varepsilon}}=\underbrace{\frac{q(r)}{r}}_{1-d} \mathbf{C}: \boldsymbol{\varepsilon}=(1-d) \mathbf{C}: \boldsymbol{\varepsilon}
$$

where $d=1-q(r) / r, d \in[0,1]$, is the continuum damage variable. The kinetic equations defining the evolution of the internal variables are described by:

$$
\begin{aligned}
& \dot{q}=H \dot{r} ; \quad \dot{r}=\gamma \\
& \left.q(t)\right|_{t=0}=q_{0}=\frac{\sigma_{0}}{\sqrt{E}}>0 ;\left.\quad r(t)\right|_{t=0}=q_{0}>0
\end{aligned}
$$

where $\sigma_{0}$ is the uniaxial peak stress and the dimensionless material parameter $H(r) \leqslant$ 0 stands for the continuum softening modulus of the model and defines the slope of the curve $q=q(r)$. Then, the loading-unloading conditions:

$$
\begin{aligned}
& \gamma \geqslant 0 ; \quad F_{\sigma}(\sigma, q) \leqslant 0 ; \quad \gamma F_{\sigma}=0 \\
& F_{\sigma}(\sigma, q)=\sqrt{\sigma: C^{-1}: \sigma}-q
\end{aligned}
$$

determine a monotonous increase of $r$, and decrease of $q$, along the pseudo-time t. In Equation (4) $F_{\sigma}$ is interpreted as a damage criterion that describes an elastic domain in the generalized stress space $(\sigma, q)$. If a linear softening law is adopted, as it is shown in Figure 1 (left), the stiffness of the material point response (tangent constitutive modulus) during a monotonic one-dimensional loading process, i.e. the $\sigma-\varepsilon$ curve slope, is given by $H E$ (see Higure 1, center). Finally, the internal dissipation of the model is a positive quantity: $D=1 / 2(q \dot{r}-\dot{q} r) \geqslant 0$, which assures the thermody-
namic consistency of the model.

As this material

observed in a bar subjected to traction, by resorting to a distributed cracking con-

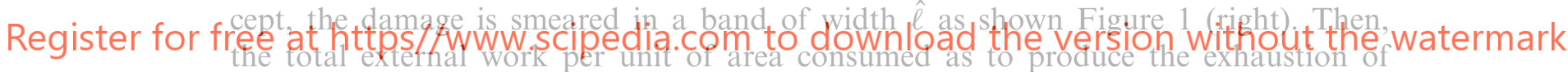

the bar loading capacity, $G_{f}$ (i.e., the fracture energy) is:

$$
G_{f}=\hat{\ell} \frac{\sigma_{0}^{2}}{2 E}\left[1+\frac{1}{|H|}\right]
$$

and assuming $|H| \ll 1$, the softening modulus $H<0$ can be characterized as:

$$
H \approx-\hat{\ell} \frac{\sigma_{0}^{2}}{2 G_{f} E}
$$
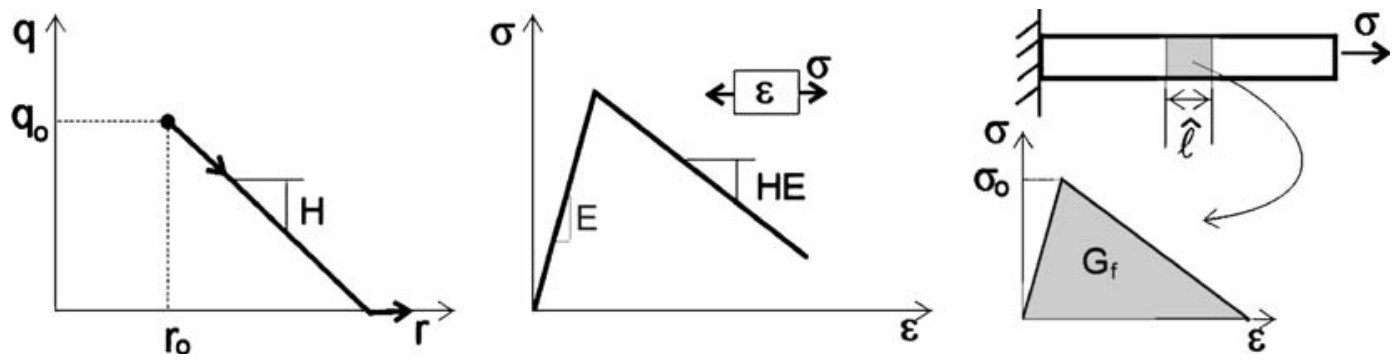

Figure 1. Softening modulus, $H$, and distributed cracking concept. 


\section{A.E. Huespe et al.}

Equation (6) supplies a physical interpretation for the softening modulus, $H$, in terms of material fracture properties.

\subsection{Strong DisCONTINUITY KINEMATICS}

Let us consider a body occupying a domain $\Omega$ in $\mathbf{R}^{3}$ and a non-smooth displacement field $\mathbf{u}(\mathbf{x})$ defined in it. A jump, across a material surface $S$, is assumed to appear in this field, which is characterized by the vector $\delta=\mathbf{u}^{+}-\mathbf{u}^{-} ; \mathbf{u}^{+}$and $\mathbf{u}^{-}$being the displacements at both sides of $S$. Here $S$ is regarded as the limit of a sequence of volumes (or slices) $S_{h}$, of bandwidth $\mathrm{h}$ and containing the surface $S: S=\lim _{h \rightarrow 0} S_{h}$ (see Figure 2). The strain field (in infinitesimal strain format) compatible with the displacement jump $\delta$ has the form (Oliver et al., 2002):

$$
\varepsilon_{S}=\varepsilon_{\Omega / S}+\lim _{h \rightarrow 0} \frac{1}{h}(\delta \otimes \mathbf{n})^{\mathrm{sym}}
$$

where $\mathbf{n}$ is the unit normal vector to $S$. From now on, subindex $(\bullet)_{S}$ and $(\bullet)_{\Omega / S}$ refer, respectively, to entities evaluated into domains $S_{h}$ and its $\Omega$-complement, $\Omega / S_{h}$. Since $\varepsilon_{\Omega / S}$ and $\delta$ are assumed bounded, Equation (7) displays the most relevant feature of the so-called strong discontinuity kinematics: the singular (unbounded) character of the strains $\varepsilon_{S}$ as $h$ tends to zero
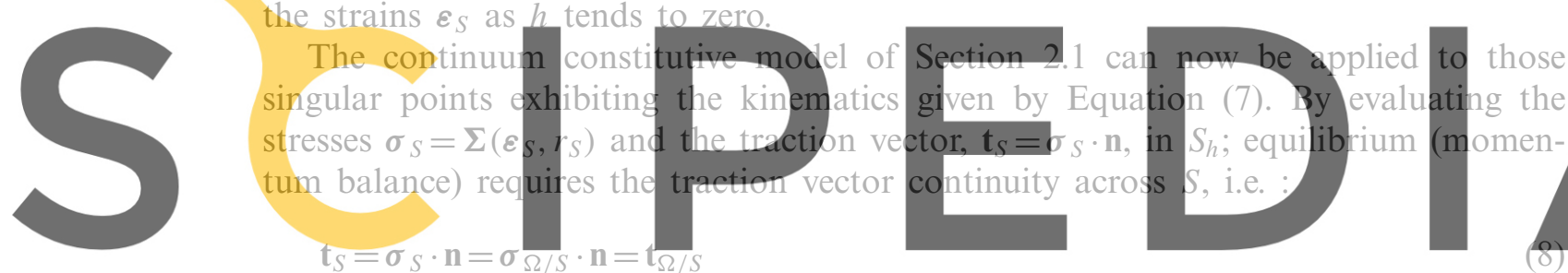

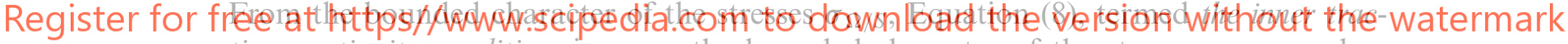
tion continuity condition, imposes the bounded character of the stress $\sigma_{S}$, even when $\varepsilon_{S}$ is a singular measure. This condition requires redefining the softening modulus in Equation (3), (see (Oliver et al., 2002)):

$$
H=h \bar{H}_{S}
$$

which is the so called continuum softening modulus regularization. Observe that, by combining Equations (9) and (6) for $\hat{\ell}=h$ one obtains (for a linear softening rule):

$$
\bar{H}_{S}=\frac{H}{h}=-\frac{\sigma_{0}^{2}}{2 G_{f} E}
$$

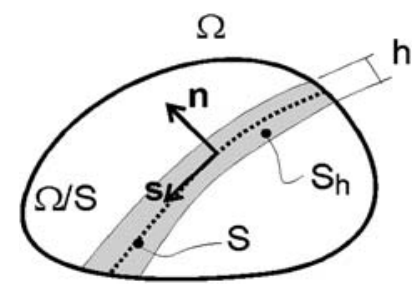

Figure 2. Discontinuous displacement field. 
which provides the physical meaning of the intrinsic softening modulus, $\bar{H}_{S}$, as a fracture material property.

A very relevant result from this regularization is that the original continuum constitutive model degenerates in a traction-displacement jump law, that is fulfilled at the discontinuity interface $S_{h}$ (see (Oliver, 2000)), according to:

$$
\mathbf{t}_{S}=\sigma_{S} \cdot \mathbf{n}=\boldsymbol{\Sigma}\left(\boldsymbol{\varepsilon}_{S}(\boldsymbol{\delta}), r_{S}\right) \cdot \mathbf{n}=\boldsymbol{\Sigma}_{S, \mathbf{n}}(\boldsymbol{\delta}, r)
$$

The constitutive law (11) is normally presented in the literature as a cohesive law relating the displacement jump $\delta$ with the traction vector $\mathbf{t}_{S}$. It can be interpreted as a projection, or degeneration, of the continuum model (1)-(4) to a cohesive law at the failure interface. Therefore, it inherits some properties of the original continuum damage mode1. The use of a continuum constitutive model, like the one in Section 2.1. to reproduce the effects of the corresponding projected cohesive model (11) into the failure interface, is the most distinguishing feature of the CSDA. That cohesive model is never derived nor implemented but the results at the failure interface $S_{h}$ are the same than if this had been done. As a counterpart, in the CSDA the volume dissipative effects in the material bulk, and the transition from continuum to discontinuum (the fracture process zone) (Bazant and Planas, 1998) can be easier and more naturally captured from the continuum format of the constitutive model. This, and other con-
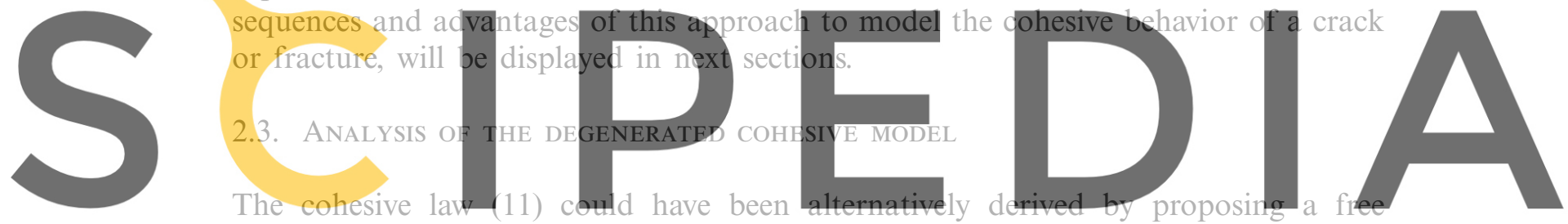

energy function per unit of area of $S, \varphi_{S}$ (surface density energy). Let us con-

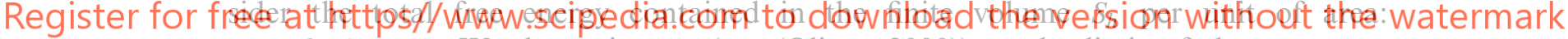

$\Phi_{h}=h \varphi\left(\varepsilon_{S}, r_{S}\right)$. We determine $\varphi_{S}$ (see $($ Oliver, 2000)) as the limit of the sequence: $\varphi_{S}(\delta, \alpha)=\lim _{h \rightarrow 0} \Phi_{h}\left(\varepsilon_{S}(\delta), r_{S}\right)$, which for the function in Equation (1), taking into account the strong discontinuity kinematics in Equation (7), results:

$$
\begin{aligned}
& \varphi_{S}(\boldsymbol{\delta}, \alpha)=\frac{1}{2} \frac{q(\alpha)}{\alpha}\left[\boldsymbol{\delta} \cdot \mathbf{Q}^{e} \cdot \boldsymbol{\delta}\right]=\frac{1}{2} \frac{q(\alpha)}{\alpha}\left[(\lambda+2 \mu) \delta_{n}^{2}+\mu \delta_{s}^{2}\right] \\
& \mathbf{Q}^{e}=\mathbf{n} \cdot \mathbf{C} \cdot \mathbf{n}=(\lambda+\mu) \mathbf{n} \otimes \mathbf{n}+\mu \mathbf{1}
\end{aligned}
$$

where $\delta_{n}=\boldsymbol{\delta} \cdot \mathbf{n}$ and $\delta_{s}=\boldsymbol{\delta} \cdot \mathbf{s}$ are, respectively, the normal and tangential components of the displacement jump, see Figure 2, and the second order tensor, $\mathbf{Q}^{e}$, is the so called elastic acoustic tensor (Willam and Sobh, 1987). The internal variable $q$ is the same than the one defined in Equation (3) for the continuum model, and $\alpha=$ $\lim _{h \rightarrow 0} h r_{S}$ is the regularized (bounded) counterpart of the strain-like internal variable $r_{S}$ (we recall that the strain field $\boldsymbol{\varepsilon}_{S}$ and the corresponding internal strain-like variable $r_{S}$ are unbounded measures of order $O(1 / h)$ ). Similarly to the continuum model case, the free energy $\varphi_{S}$ is used as a potential for determining the traction vector $\mathbf{t}_{S}$ :

$$
\mathbf{t}_{S}=\frac{\partial \varphi_{S}(\boldsymbol{\delta}, \alpha)}{\partial \boldsymbol{\delta}}=\underbrace{\frac{q}{\alpha}}_{1-\omega} \mathbf{Q}^{e} \cdot \boldsymbol{\delta}=(1-\omega) \mathbf{Q}^{e} \cdot \boldsymbol{\delta}
$$



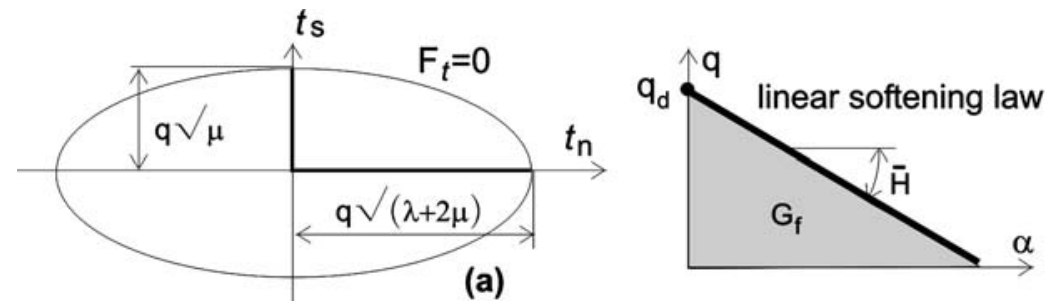

(b)

Figure 3. Cohesive degenerated model. (a) Criterion for activation of the dissipative mechanism in the space of generalized forces; (b) Softening response of the traction-separation law.

where $\omega=1-q(\alpha) / \alpha,(\omega \in(-\infty, 0])$, is a discrete damage variable. The evolution equations for the internal variables are then defined as:

$$
\begin{aligned}
& \dot{q}=\bar{H}_{S} \dot{\alpha} ; \quad \dot{\alpha}=\hat{\gamma} \\
& \left.q(t)\right|_{t=t_{d}}=q_{d}=\frac{\sigma_{d}}{\sqrt{E}}>0 ;\left.\quad \alpha(t)\right|_{t=t_{d}}=0
\end{aligned}
$$

where $t_{d}$ is the time of onset of local damage (cracking). The loading-unloading conditions read:
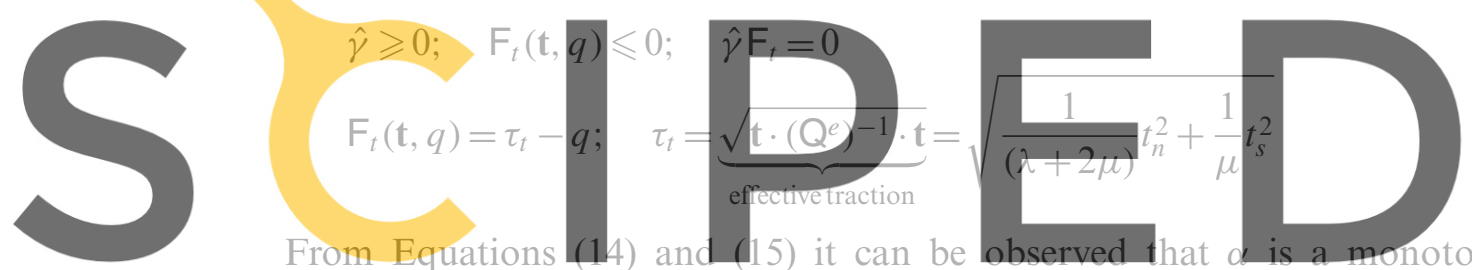

ing function in time $(\dot{\alpha} \geqslant 0)$. Now, $F_{t}$ is a damage criterion defined in the space of

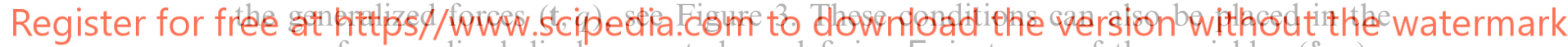

space of generalized displacements by redefining $F_{t}$ in terms of the variables $(\delta, \alpha)$ :

$$
\begin{aligned}
& \hat{\gamma} \geqslant 0 ; \quad \boldsymbol{F}_{\delta}(\boldsymbol{\delta}, \alpha) \leqslant 0 ; \quad \hat{\gamma} \mathrm{F}_{\delta}=0 \\
& \mathbf{F}_{\delta}(\boldsymbol{\delta}, \alpha)=\tau_{\delta}-\alpha \\
& \tau_{\delta}=\underbrace{\sqrt{\boldsymbol{\delta} \cdot \mathbf{Q}^{e} \cdot \boldsymbol{\delta}}}_{\text {effective crack opening }}=\sqrt{(\lambda+2 \mu) \delta_{n}^{2}+\mu \delta_{s}^{2}}
\end{aligned}
$$

From Equation (16), and the aforementioned condition $\dot{\alpha} \geqslant 0, \alpha$ can be interpreted as the maximum effective crack opening along the loading process history.

In a complete process of crack decohesion, the total energy dissipated at the discontinuity surface, $W_{S}$, results:

$$
\begin{aligned}
& W_{S}=\int_{S}\left(\int_{t_{d}}^{\infty} \mathbf{t} \cdot \dot{\boldsymbol{\delta}} \mathrm{d} t\right) \mathrm{d} S=\int_{S} \underbrace{\left(\int_{0}^{\infty} q \cdot d \alpha\right)}_{G_{f}} \mathrm{~d} S \\
& G_{f}=\int_{0}^{\infty} q \cdot \mathrm{d} \alpha=\frac{1}{2} \frac{q_{d}^{2}}{\bar{H}}=\frac{1}{2} \frac{\sigma_{d}^{2}}{\bar{H} E}
\end{aligned}
$$


which matches the classical concept of Fracture Energy in cohesive models as the consumed energy per unit of the crack area (Bazant and Planas 1998) (see Figure $3 b)$.

Remark 1. Unlike in the crack band model of Bazant (1983), in the CSDA, the softening modulus is not regularized in terms of a characteristic length, $\ell_{c h}$, playing the role of a material parameter. Here, though the continuum strain softening regularization (9) introduces the length $(h)$ in the dimension of the regularized softening parameter $\bar{H}$, this length is not a physical property, but a computational regularization parameter as small as permitted by the machine precision.

Remark 2. The cohesive model induced by Equations (12)-(16) belongs to a subclass of the family of cohesive models described by Ortiz et al. in (Oritz and Pandolfi, 1999). In that framework, the surface free energy density, $\varphi_{S}$, in Equation (12) verifies the material frame indifference requirements:

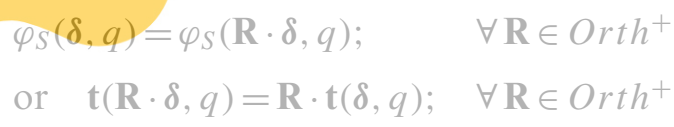

This property is inherited from the continuum free energy density in Equation (12). Furthermore, Ortiz et al. introduce an effective traction $\tau^{\mathrm{op}}=\sqrt{t_{n}^{2}+\beta^{-2} t_{s}^{2}}$
to define the crack loading criteria. The material parameter $\beta$ in Equation $(1$
the ratio between the normal $\left(t_{n}=\mathrm{t} \cdot \mathrm{m}\right)$ and tangential $\left(t_{s}=\mathbf{t} \cdot \mathbf{s}\right)$ critical traction ponents, and it is assumed characterizable by an additional experimental test. On the

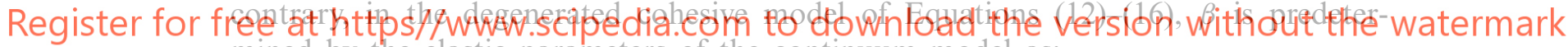
mined by the elastic parameters of the continuum model as:

$$
\beta=\sqrt{\frac{\mu}{\lambda+2 \mu}}
$$

as it can be checked from Equation (15b).

Remark 3. Prior to the crack activation, the traction-separation law $\mathbf{t}(\boldsymbol{\delta})$ induced by the CSDA is naturally inactive, i.e.: the crack remains closed $(\delta=0)$ at any material point during the pre-critical (elastic) states. Indeed, in the elastic regime, for the continuum model, the traction continuity condition $\boldsymbol{\sigma}_{S} \cdot \mathbf{n}=\boldsymbol{\sigma}_{\Omega / S} \cdot \mathbf{n}$ imposes that $\boldsymbol{\varepsilon}_{S}=$ $\boldsymbol{\varepsilon}_{\Omega / S}$ and, from Equation (7), $\boldsymbol{\delta}=0$. This makes unnecessary the introduction of any kind of dummy stiffness in the traction/separation law, $\mathbf{t}(\boldsymbol{\delta})$, to enforce the crack closure before the fracturing criterion is fulfilled. As it will be shown in Section 4, the typical oscillatory traction profile displayed by the cohesive models with high dummy stiffness (see Rots (1988), pp. 63, Simone (2004)) does not show up by using the CSDA.

Remark 4. (Time continuity of the traction-separation law): Papoulia et al. (2003) have introduced the notion of time continuity related to cohesive laws, within the 


\section{A.E. Huespe et al.}

context of interface finite element models. The authors advocate that lack of that pseudo-time continuity, in traction/separation laws, leads to numerical instabilities and loss of convergence of the iterative numerical procedure to reach the equilibrium states. This effect cannot be overcome by reducing the time step. Traction/separation laws, introduced after crack activation in an ad hoc manner, do not exhibit, in general, such time continuity, which should be enforced via the so-called encoding procedure (Papoulia et al., 2003). On the contrary, in the CSDA any selected continuum constitutive model, $\sigma=\Sigma(\boldsymbol{\varepsilon}(\mathbf{t}))$, like the one in Equations (1)-(4), is a pseudo-time continuous function and, therefore, the map $\boldsymbol{t}_{\boldsymbol{s}} \mapsto \sigma_{\mathrm{S}}(t) \cdot \mathbf{n}$ is a time continuous projection, even at the crack activation time $t_{\text {loc }}$ :

$$
\left.\mathbf{t}_{\mathrm{S}}(t)\right|_{t=t_{\mathrm{loc}}}=\lim _{t \rightarrow t_{\mathrm{loc}}}\left(\sigma_{\mathrm{S}}(t) \cdot \mathbf{n}\right)
$$

Therefore, although the degenerated continuum model in Equations (12)-(16) is a rigid-model (i.e.: the initial secant stiffness in Equation (13) is infinity) and Equation (13) is undetermined at $t=t_{\mathrm{loc}}$ (being $\delta\left(t_{\mathrm{loc}}\right)=\mathbf{0}$ and $\left.\alpha\left(t_{\mathrm{loc}}\right)=0\right)$, Equation (21) defines uniquely the traction vector, $\mathbf{t}_{S}$, at $t=t_{\mathrm{loc}}$, which removes that indetermination.

\section{Modeling the fracture process zone}
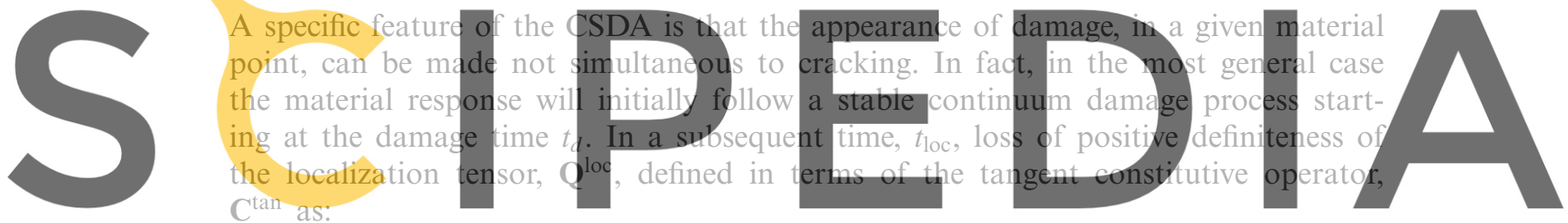

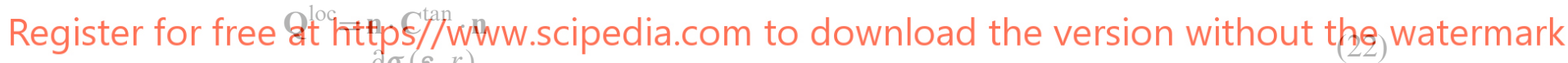
$\mathrm{C}^{\mathrm{tan}}=\frac{\partial \sigma(\varepsilon, r)}{\partial \varepsilon}$

signals the onset of the material instability (Oliver and Huespe, 2004) and the formation of the displacement discontinuity that characterizes the crack. The delay of $t_{\text {loc }}$ in front of $t_{d}$ depends, for a given material model, on the material properties and the stress state. For a given stress state, the less negative is the softening modulus, $H$ in Equation (3), the larger is the interval $\left[t_{d}, t_{\mathrm{loc}}\right]$. Therefore, from a phenomenological approach to crack modeling, one can take advantage of this fact to model the amount of volumetric dissipation in front of the crack tip and, indirectly, the size and influence of the fracture process zone. This is sketched in Figure 4: the initial value of the continuum softening modulus, a material property $H_{\text {con }}$, rules the stable damage production at the considered point (see Figure 4a). Material points in this stage, in front of the crack tip, are displayed in the grey zone in Figure $4 \mathrm{~b}$, between points $\mathbf{D}$ (standing for onset of damage) and $\mathbf{L}$ (standing for onset of localization). The length $\ell_{d}$ of this stable damaged zone is governed by the value $H_{\text {con }}$ as said before. As, at time $t_{\text {loc }}$, material instability is detected the continuum softening modulus is no longer determined as a material property but regularized from the intrinsic softening modulus $\bar{H}$ (in turn depending on the fracture energy) according to Equation (9), see Figure 4a. At the same time, the strong discontinuity kinematics (7) is 

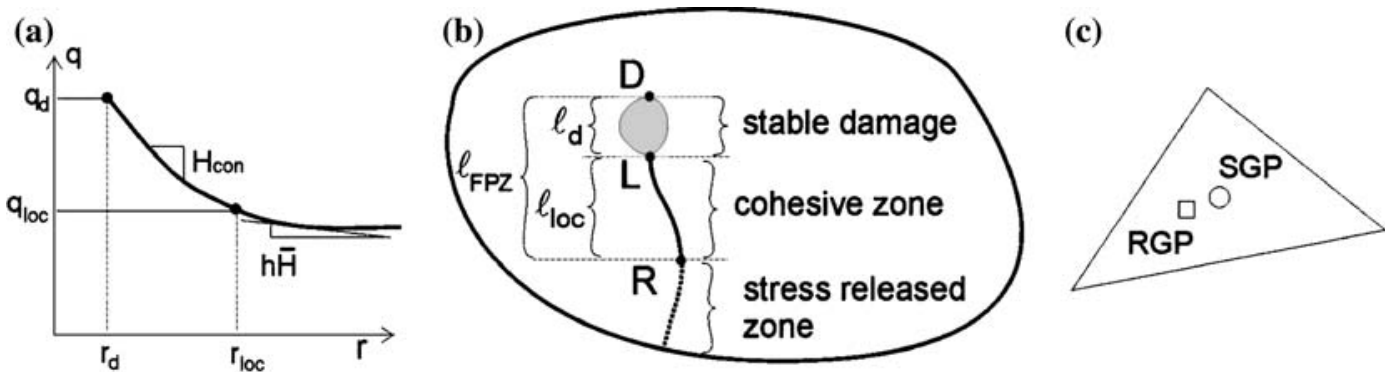

Figure 4. (a) Softening rule, (b) Generalized fracture process zone and (c) Regular (RGP) and singular (SGP) Gauss Points in a finite element with embedded discontinuity.

activated, this ensuring fulfillment of the degenerated traction/separation law and the subsequent correct dissipation of the fracture energy $G_{f}$ up to the complete stress release. Points in this stage are signaled, in Figure $4 \mathrm{~b}$, as the cohesive zone between points $\mathbf{L}$ (standing for onset of localization) and $\mathbf{R}$ (standing for stress release). Both, the stable damage and the cohesive zones, constitute the generalization, in a CSDA setting, of the fracture process zone (FPZ), classically considered in non-linear fracture mechanics (Bazant and Planas, 1998), as the locus of material points where the fracture is processed by dissipation mechanisms. As it will be shown in Section 4.1,

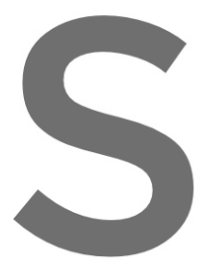

the total size of th

of fracture energy

sponding dissipatio

the size of the cohesive zone, $\ell_{100}$, can be expressed as:

$\ell_{\mathrm{loc}}\left(G_{f}, H_{\mathrm{con}}\right)=\ell_{F P Z}\left(G_{f}\right)-\ell_{d}\left(H_{\mathrm{con}}, G_{f}\right.$
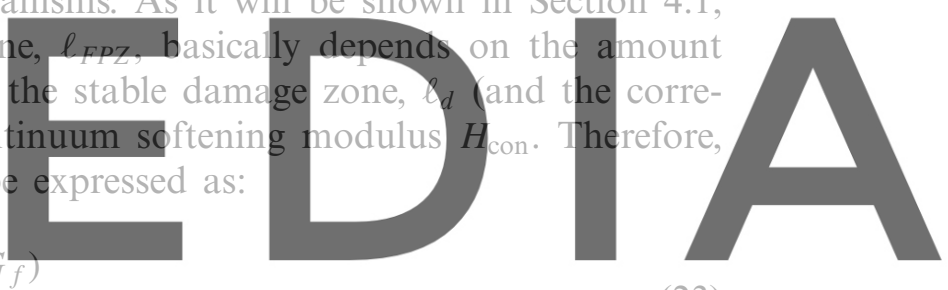

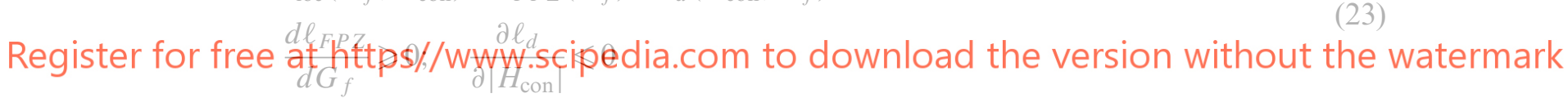

In this way the CSDA provides a setting to model the transition from continuum to discontinuum and to characterize the complete fracture process zone.

\section{Representative numerical simulations and assessment of the CSDA}

The CSDA described in previous sections can be readily implemented in numerical modeling scenarios using an embedded strong discontinuity finite element technique (Oliver et al., 2002). The distinguishing features of finite elements with embedded discontinuities with respect to standard finite elements are essentially two (Oliver and Huespe, 2004): (a) the standard deformation modes are supplemented with additional deformation modes, which reproduce the strong discontinuity kinematics in Equation (7), and (b) besides the standard integration points (the "regular Gauss points", RGP in Figure 4c) each element is equipped with an additional Gauss point (the "singular Gauss point" SGP in Figure 4c) where the behavior of the discontinuity interface, $S$, in terms of the kinematics (7) and the softening regularization (9) is reproduced. In addition, the inner traction continuity Equation (8) is enforced in terms of the stresses at the regular and singular sampling points, this providing an 


\section{A.E. Huespe et al.}

additional equation that resolves the elemental displacement jump. In elastic regimes, this equation is trivially fulfilled with the contributions of the standard deformation modes and, then, the enriching discontinuous displacement modes are not activated.

In the following sections, some well-known, and widely studied, crack propagation problems are modeled in the CSDA context. The simulation is here addressed to present the distinguishing features supplied by the degenerated cohesive model to the numerical solution. In order to reproduce the different tensile and compressive fracture behavior of quasi-brittle materials, like concrete, the continuum damage model of Section 2.1 is slightly modified in the following sense: the damage criterion of equation (4) is switched to:

$$
\begin{aligned}
& \boldsymbol{F}_{\sigma}(\boldsymbol{\sigma}, q)=\sqrt{\boldsymbol{\sigma}^{+}: \mathbf{C}^{-1}: \boldsymbol{\sigma}}-q \\
& \sigma^{+}=\sum_{i=1}^{i=3}\left\langle\sigma_{i}\right\rangle \mathrm{p}_{i} \otimes \mathrm{p}_{i}
\end{aligned}
$$

where $\sigma^{+}$stands for the positive part of the stress tensor $\sigma, \sigma_{i}$ and $\mathbf{p}_{i}$ stand, respectively, for the eigenvalues (principal stresses) and eigenvectors of $\sigma$, and $\left\langle\sigma_{i}\right\rangle$ is the positive part of $\sigma_{i}\left(\left\langle\sigma_{i}\right\rangle=\sigma_{i}\right.$ for $\sigma_{i}>0$ and $\left\langle\sigma_{i}\right\rangle=0$ otherwise $)$. With this modification, damage is restricted to tension dominated states so that the model will be termed

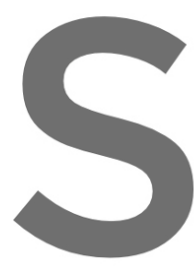
tension damage model. Th
then automatically inherit
law without necessity of an
4.1. THREE-POIN N мотсHED
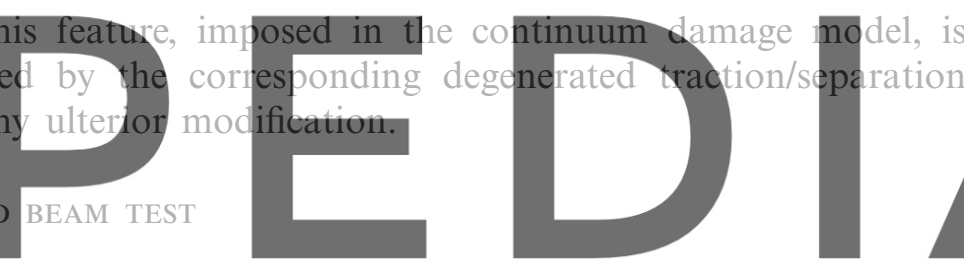

The crack propagation problem in a notched concrete beam supported on three

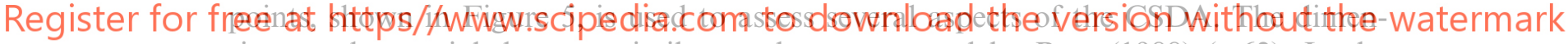
sions and material data are similar to those presented by Rots (1988) (p.62). In the present numerical study, a rather fine finite element mesh of linear triangles, with 25 elements discretizing the domain extending from the notch tip to the load application point $(\approx 80 \mathrm{~mm})$, is used. In these elements, the main phenomena involved in the crack propagation are going to be captured. The continuum constitutive model is the tension damage model, introduced above, specified for the plane stress case and equipped with a linear softening law. Although tracking procedures for automatic determination of the crack propagation path are available (Oliver et al., 2004; Oliver and Huespe, 2004), here that path has been artificially predetermined as the vertical line starting from the notch tip in order to make the results independent of the determination of that crack path.

\subsubsection{Assessment of time continuity}

Figure 5 shows the evolution, in terms of the pseudo-time $u_{y}$ (the monotonically increasing vertical displacement of the load application point), of the normal and tangential components of the traction vector $\left(\mathbf{t}_{S}=\sigma_{S} \cdot \mathbf{n}\right)$ at four different points of the crack path (points $\mathrm{A}, \mathrm{B}, \mathrm{C}$ and $\mathrm{D}$ at a distance $\zeta$ from the notch tip). There it can be observed that both components of $\mathbf{t}_{S}$ are continuous all along the 
(a)
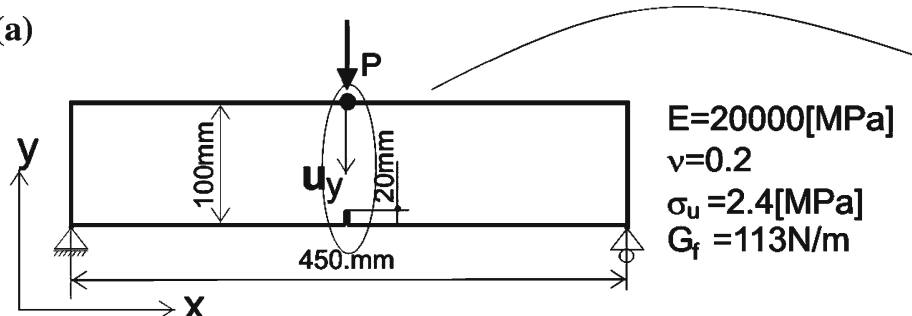

(b)

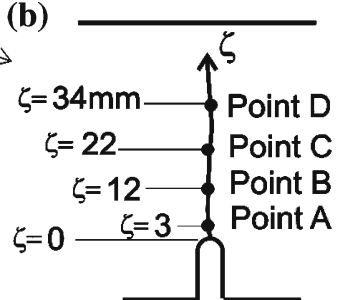

(c) Traction normal component $t_{n}[\mathrm{MPa}]$
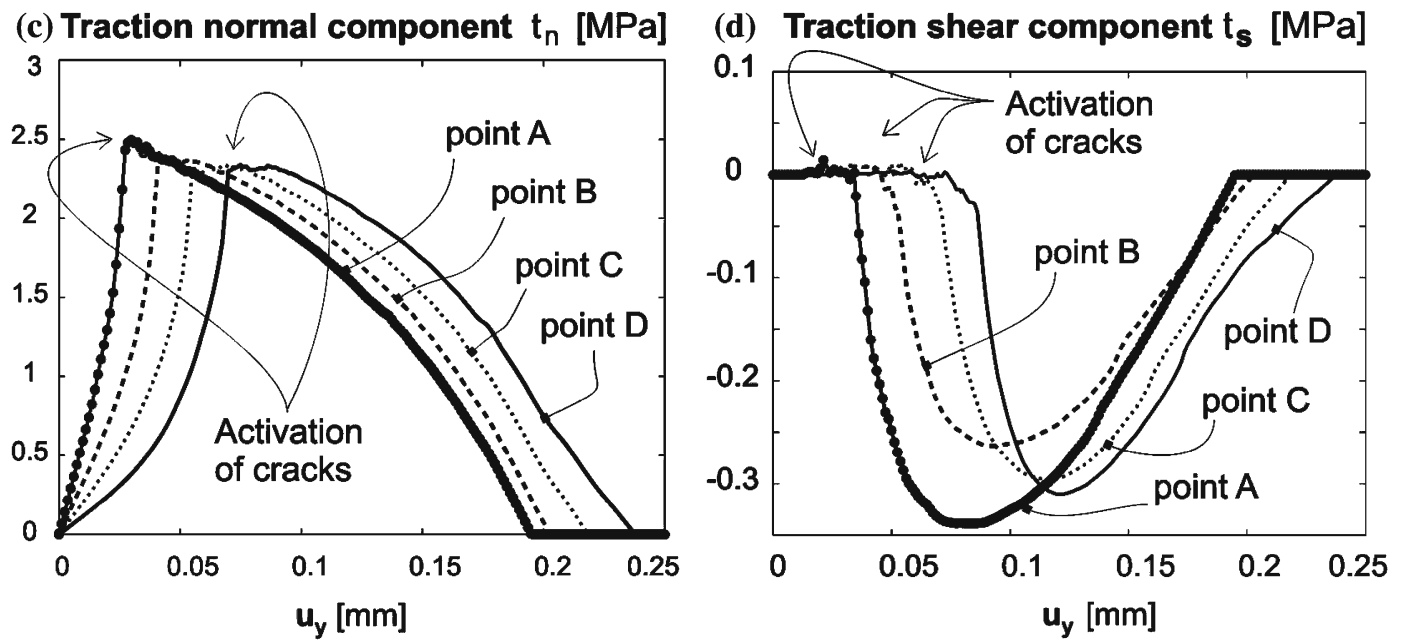

Figure 5. Notched three-point beam test: (a) Problem description, (b) Selected points in the crack path, (c) and (d) Evolution of the normal and tangential traction components.

time, irrespective of the transition from the continuous to crack activated states. This assesses the aforementioned time continuity of the degenerated traction-separation law (see Remark 4).

\subsubsection{Action-response smoothness}

Figure 6a shows the pseudo-time evolution of the applied force (action-response curve). In Figure 6b, spatial profiles of the normal component of the traction, $t_{n}$, along the crack path, as a function of the coordinate $\zeta$, for the different times signaled in Figure 6a, are presented. Unlike what is mentioned in the literature about the oscillatory behavior of the traction components when using cohesive models equipped with large dummy elastic stiffness (Rots (1988), Alfano et al. (2001), Planas et al. (2004)), in the results provided by the CSDA that oscillatory behavior is not observed. The profile labeled $t_{1}$, corresponds to an elastic response during the loading process (at time $t_{1}$ all elements are in the elastic range) and, as expected, is smooth. The same smooth response can be observed, in Figure 6b, for all the profiles at subsequent times, even when they involve material points in softening range and also in released stress states (profiles $t_{2}$ to $t_{5}$ ). The reason for this is that the traction-separation law (13), induced at the crack by the CSDA via the projection of the continuum constitutive Equation (2), though exhibiting an initial infinite secant stiffness (which would correspond to an infinite value of the dummy stiffness in the cohesive model) is integrated in its continuum format in Equation (2)-(4), without involving that singular initial stiffness (see Remark 3). 
(a)

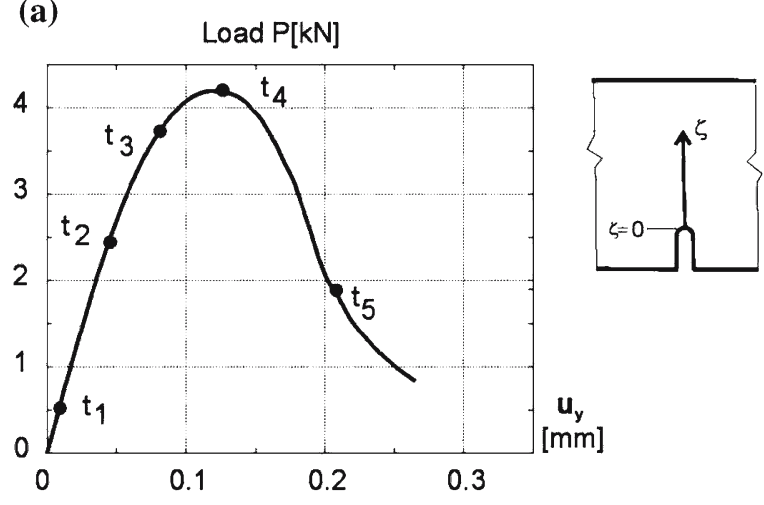

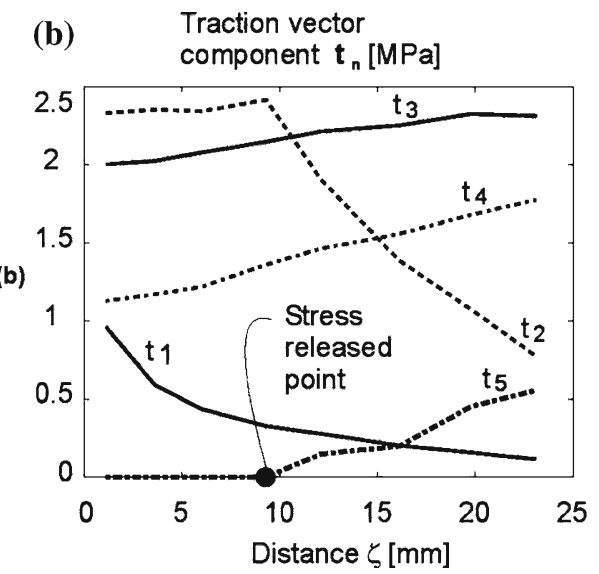

Figure 6. Notched three-point beam test: a) Force-displacement curve, b) Profiles of the normal traction component distribution at different stages of the analysis.

\subsubsection{Stress mismatch}

Another observed problem in classical cohesive models, pointed out by Elices et al. (2002) and Planas et al. (2003) and termed cohesive model mismatch, is related to the distribution and properties of the stress states in bulk points at the immediate neighborhood of the crack tip.

These authors observed that, using classical cohesive models and a propagation criterion based on the maximum principal stress, the directions of maximum principal stresses in those points becomes parallel to the crack path and their magnitude are greater than the ultimate tensile stress $\boldsymbol{\sigma}_{u}$.

Is in this context that the numerical solution in Figure 7, obtained in a CSDA format, is analyzed regarding the role of the initial continuum softening modulus, $H_{\text {con }}$, described in Section 3.

The continuum format of the CSDA allows accounting for the energy dissipation during the loading process in points undergoing stable softening. This leads to the stress state presented, in terms of the principal stress vectors in Figure 7, as the crack propagation length reaches $17 \%(\approx 17 \mathrm{~mm})$ of the total beam height. There, it can be observed that the stress state at the crack tip neighborhood is, approximately, hydrostatic (both principal stresses are similar) in agreement with the literature. In addition, near the consolidated crack, the largest principal stress holds orthogonal to the crack path and smaller than the peak stress. The stress mismatch is, then, not present in the results. It is also noticed in Figure 7 the existence of a singular point $(\sigma=0)$ nearby the crack tip and in front of it. The appearance of this singular point (all the directions are principal) could be the source of the reported difficulties (see (Mosler and Moschke, 2003) for instance) when tracking algorithms and too coarse finite element meshes are used.

\subsubsection{Stress field smoothness}

In Figure 8 the stress fields for two limit cases are shown. Figure 8a corresponds to a very small stable damage zone achieved by using a very large (negative) value of the initial softening modulus, $H_{\text {con }}$, whereas Figure $8 \mathrm{~b}$ corresponds to a large 


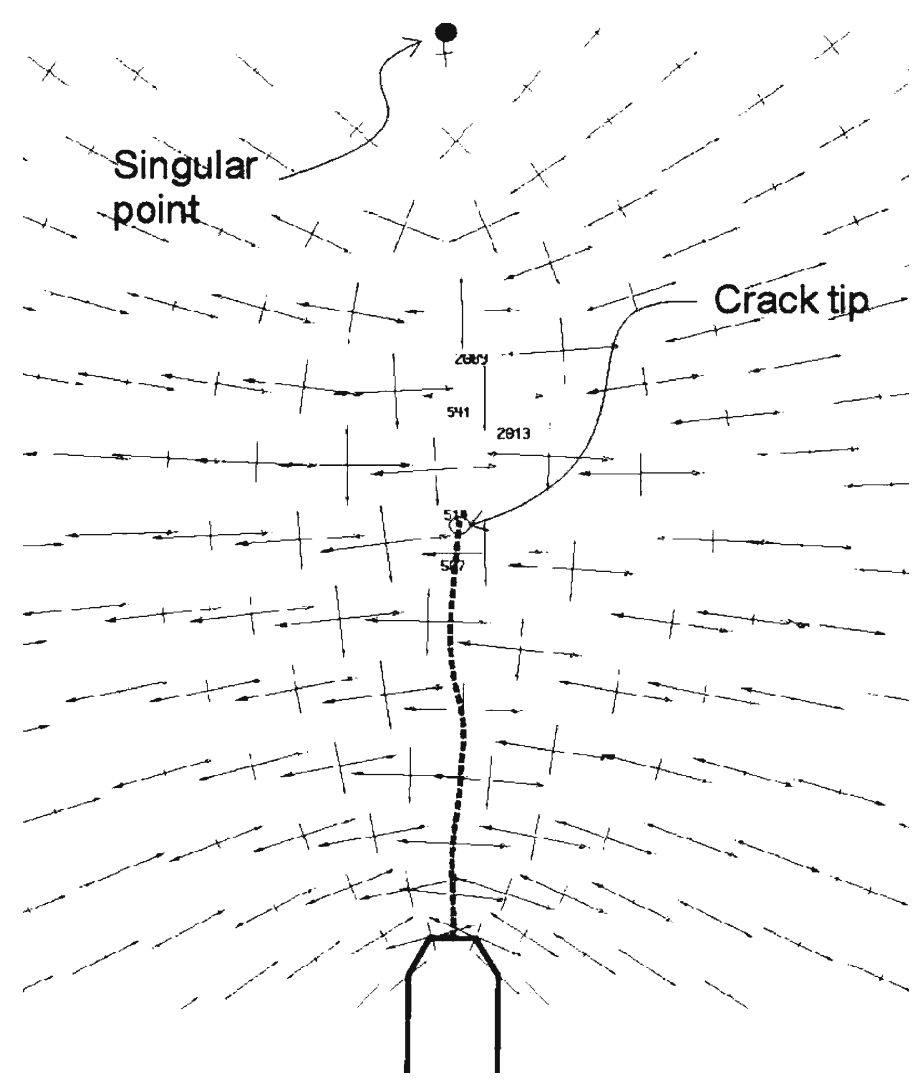

Figure 7. Notched three-point beam test: Principal stress field in the neighborhood of the crack.

stable damage zone achieved by using an almost null (but still negative) initial softening modulus $H_{\text {con }}$. Therefore, results in Figure 8a would match the ones obtained with a classical cohesive model where the behavior in the bulk is assumed totally elastic.

The figures also display, in a grey scale, the elements exhibiting a maximum principal stress larger than the tensile material strength $\sigma_{u}$. Comparison of both figures clearly shows the beneficial effects of the bulk dissipation mechanisms in the smooth character and consistency of the resulting stress fields.

\subsubsection{Fracture process zone}

Let us focus on the role played by the initial continuum softening modulus, $H_{\text {con }}$ of the softening rule in Figure $4 \mathrm{a}$, and its influence on the fracture process zone structure. In order to allow a clearer and more complete development of that fracture process zone, the analysis is carried out on a beam higher than the preceding one (the beam height in Figure $5 \mathrm{a}$ is now changed to $0.24 \mathrm{~m}$ ).

Curves in Figure 9a show the dependence of the fracture process zone length, $\ell_{F P Z}$, on the fracture energy, $G_{f}$, as a function of the material characteristic length parameter $\left(\ell_{c h}=E G_{f} / \sigma_{u}^{2}\right)$ characterizing the material brittleness (see Elices et al. (2002)). In the figure it can be observed that $\ell_{F P Z}$ depends clearly on $G_{f}$ but it barely changes with those substantial changes of $H_{\text {con }}$. Thus, the experiment assesses 
(a)

\section{Crack tip element}

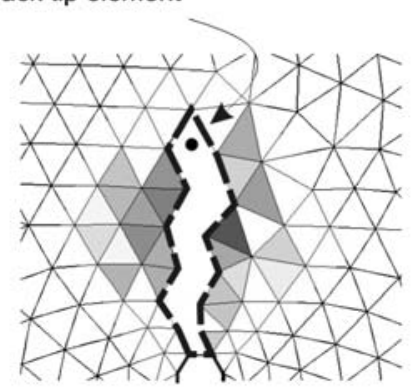

Elastic bulk response

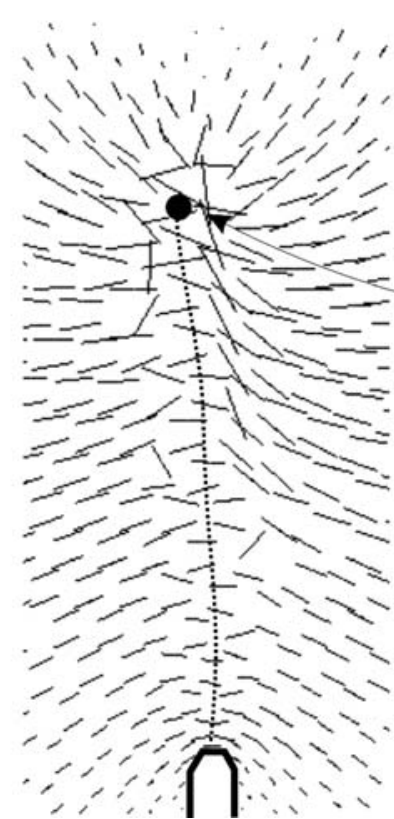

(⿸丆口

(b)

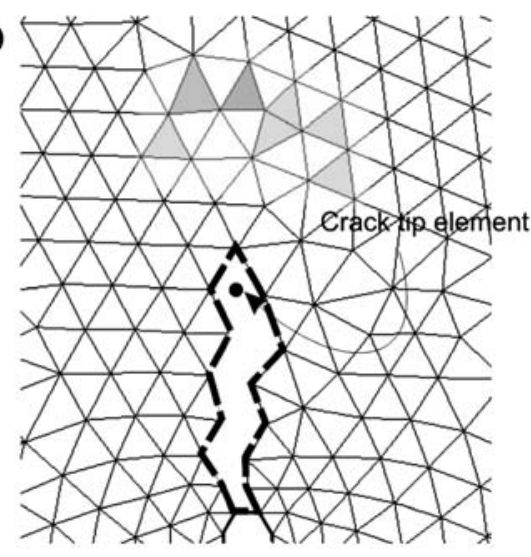

Energy dissipation outside the crack

Maximum Principal stress [MPa] (material strength $\sigma_{u}=2.4 \mathrm{MPa}$ )
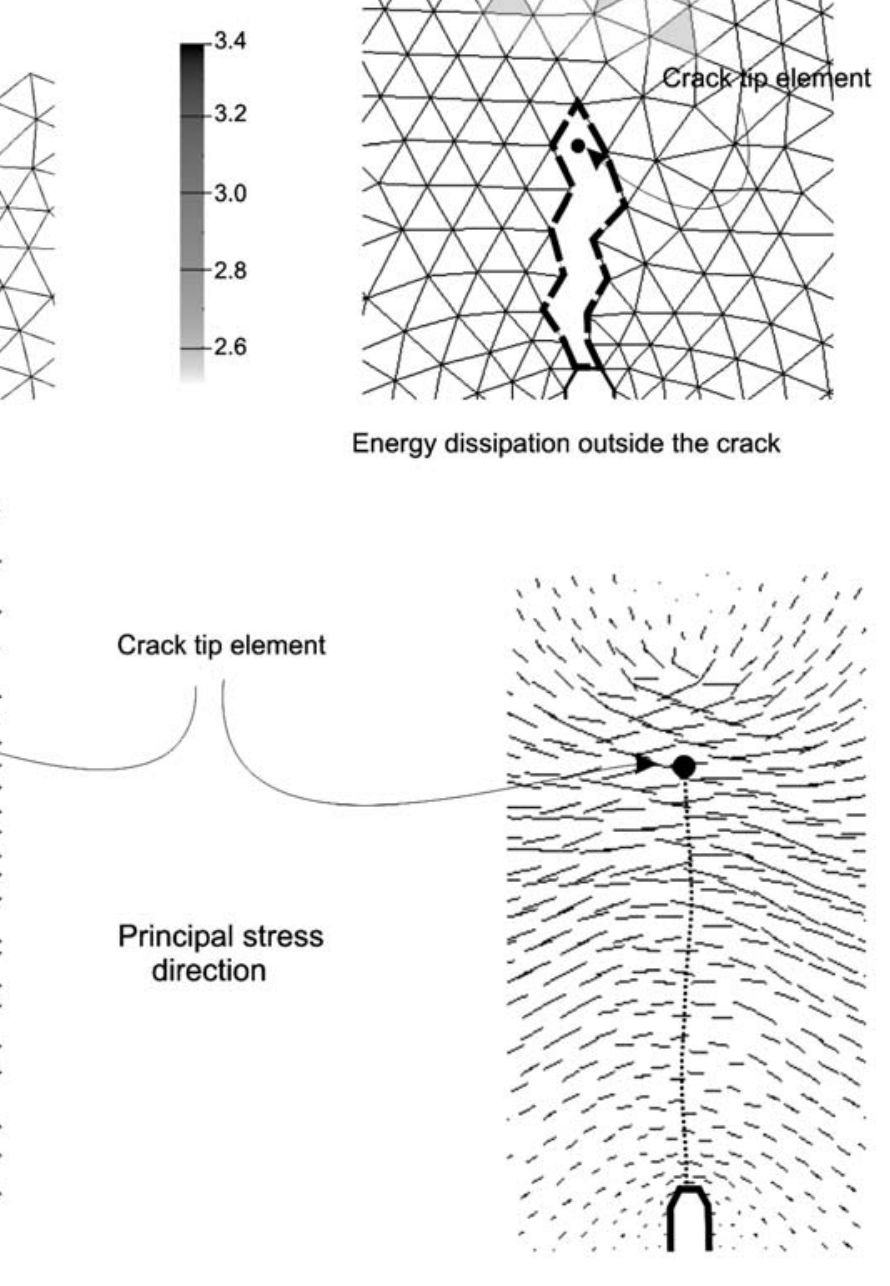

Figure 8. Notched three-point beam test. Principal stresses and elements (in a grey scale) with a principal stress larger than the material peak strength. (a) Null stable damage zone (b) Large stable damage zone.

the exclusive dependence, stated in Equation (23), of the total length of the fracture process zone on the fracture energy, $G_{f}$.

In Figure $9 \mathrm{~b}$, it is shown the dependence of the stable damage zone size, $\ell_{d}$, with the $H_{\text {con }}$ and $G_{f}$ values. Results are parameterized in terms of the normalized length, $\chi,\left(\chi=-H_{\text {con }} \ell_{c h}\right)$. Plots of curves $\ell_{d}(\chi)$, for different values of $\ell_{c h}\left(G_{f}\right)$, are then presented. In agreement to the assertion in Bazant (1996), about the changing length of the micro cracking zone in front of the crack tip, the numerical simulations show a similar behavior. Therefore, all lengths in Figure $9 \mathrm{~b}$ have been measured at the time as stresses are completely released at the notch tip (complete stress release). It can be observed that, for a given value of $G_{f}$, increasing values of $\left|H_{\text {con }}\right|$, diminish the stable damage zone size $\ell_{d}$ as stated in Equation (23) (the lower bound is, approximately, the size 

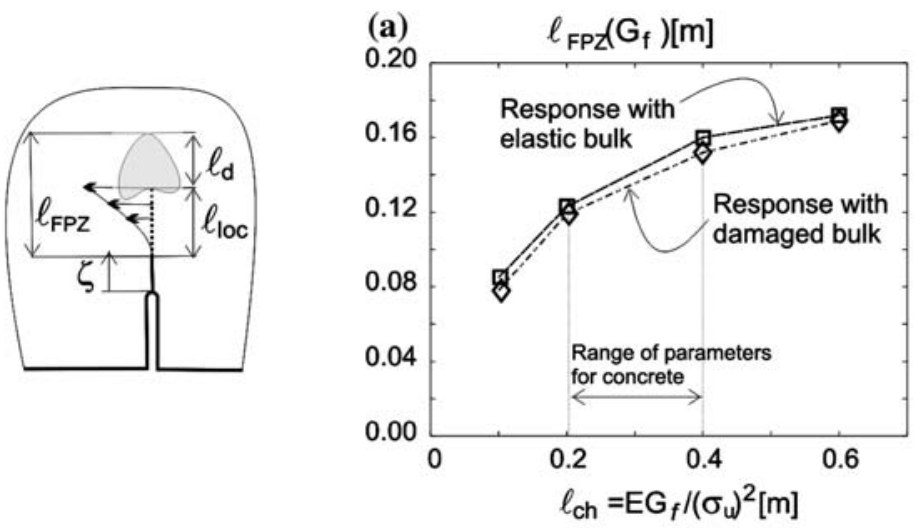

(c) Response with elastic bulk

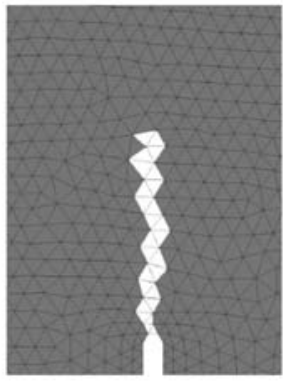

(b)

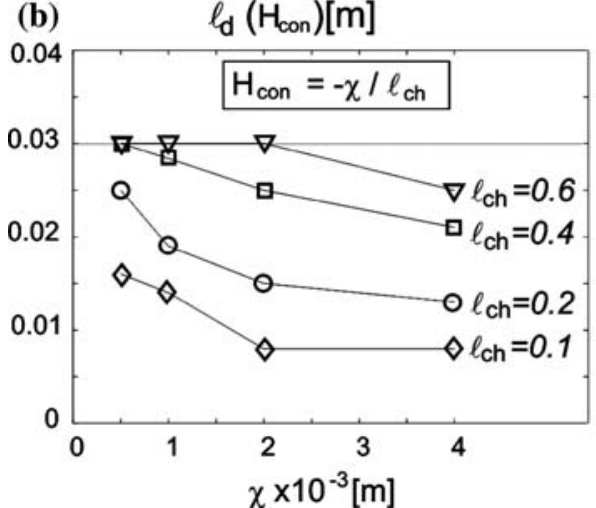

(d)

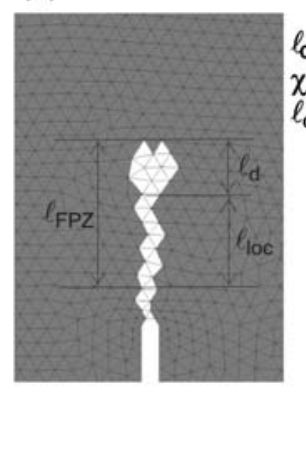

(e)

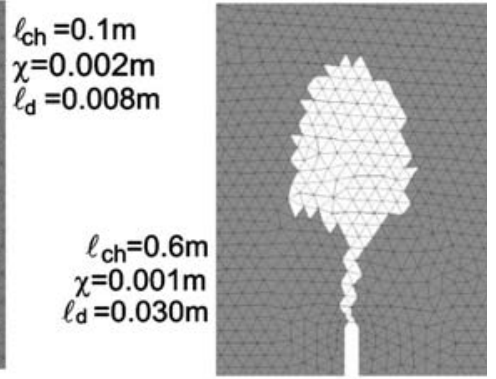

Figure 9. Fracture process zone development in a three-point bending test of a notched beam: (a) Plots of the fracture process zone lengths, $\ell_{F P Z}$, versus the material characteristic length $\ell_{c h}$; (b) Stable damage zone length, $\ell_{d}$, versus the initial softening modulus $H_{\text {con }}$; (c,d,e) Snap shots of the FPZ (dissipating finite elements in light grey) for different cases.

of one finite element $0.004 \div 0.007 \mathrm{~m}$ ). Typical developments of the FPZ, for different material parameter values, are displayed in Figure 9c-e (finite elements undergoing dissipation are plotted in light grey color). There, the stable damage zone (round shaped in front the crack tip) can be clearly differentiated from the remaining of the FPZ.

\subsection{Double Cantilever beam (DCB Test)}

Another typical example of a Mode I crack propagation problem, the double cantilever beam test (DCB) of Figure 10, has been solved considering a plain stress model (of $1 \mathrm{~m}$ width) with dimensions and material data similar to those presented in $\mathrm{Zi}$ et al. (2003). The crack propagation path has been set to the horizontal line starting at the notch tip. A structured finite element mesh, of linear triangles with $7.0 \mathrm{~mm}$ side length, has been constructed to model the cohesive zone. This mesh size is one order larger than in the mesh reported in ( $\mathrm{Zi}$ and Belytschko, 2003).

\subsubsection{Predicted peak-load values}

This test emphasizes the effects on the predicted peak loads, of the inclusion in the simulation of the stable dissipation zone via the initial continuum softening modulus, 
(a)

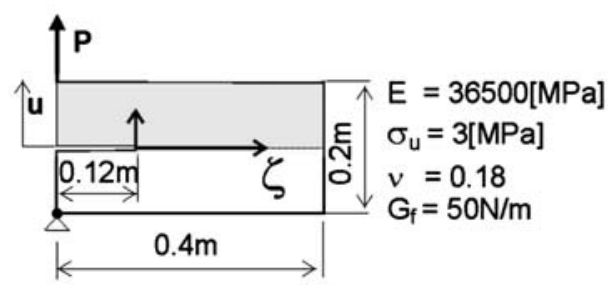

(a)
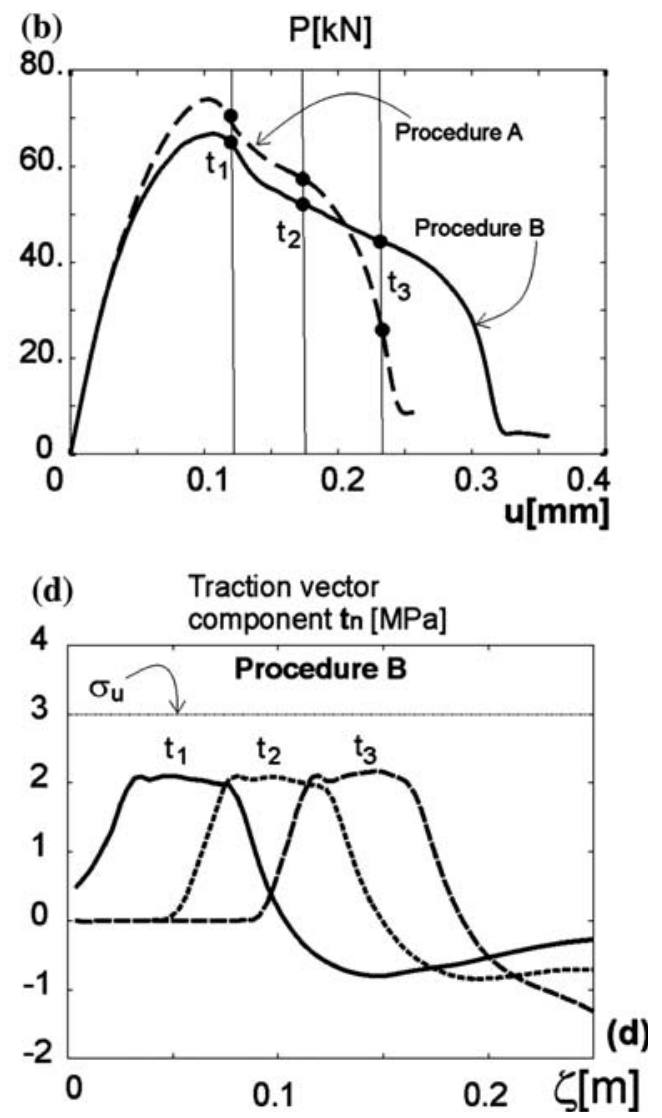

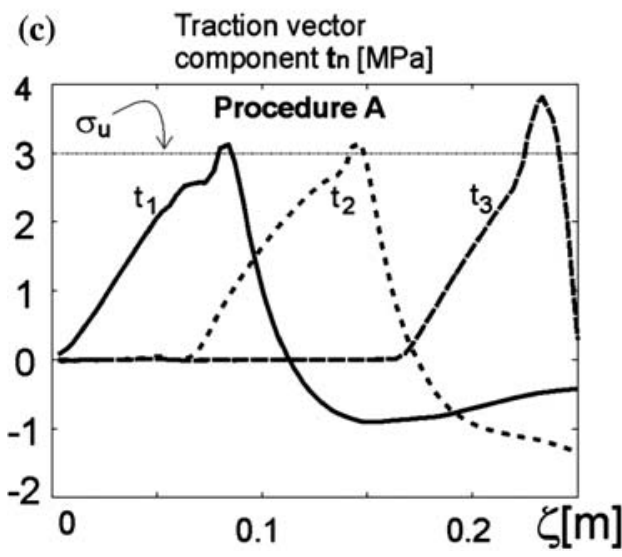

Figure 10. Double cantilever beam test. (a) Geometrical model and material data; (b) loaddisplacement (CMOD) curves; (c) and (d) normal traction component along the crack path at different times.

$H_{\text {con }}$. Like in previous cases, the problem has been solved using two different procedures. In Procedure A, an elastic response is imposed at the bulk, like in standard cohesive models, by using a very large (negative) value of the initial softening modulus, $H_{\text {con }}$ (stable dissipation zone does not exist); in Procedure $B$, bulk dissipation is admitted by specifying a softening modulus $H_{\text {con }} \approx 0$ (large stable dissipation zone). In both cases the same value for the fracture energy $G_{f}$ is considered. Figure 10b displays the corresponding force-displacement (CMOD) curves. It can be checked that allowing dissipation on the bulk has a non-negligible effect on the predicted peak load, in the sense of lowering them.

\subsubsection{Consistency of the stress profiles}

Figure $10 \mathrm{c}$ plots the distribution of the crack normal stress component, $t_{n}$, along the crack path (stress profile), at the three different times, $t_{1}, t_{2}$ and $t_{3}$, specified in the equilibrium curves of Figure 10b. Those curves computed with the Procedure $A$ (standard cohesive model) show peak values greater than the material tensile strength $\left(\sigma_{u}=3 \mathrm{MPa}\right)$. This inconsistency has also been detected by $\mathrm{Zi}$ et al. (2003) using a similar procedure. These authors claim that this unwelcome effect disappears, or diminishes, with mesh refining. Results obtained with Procedure $B$ (bulk dissipation 


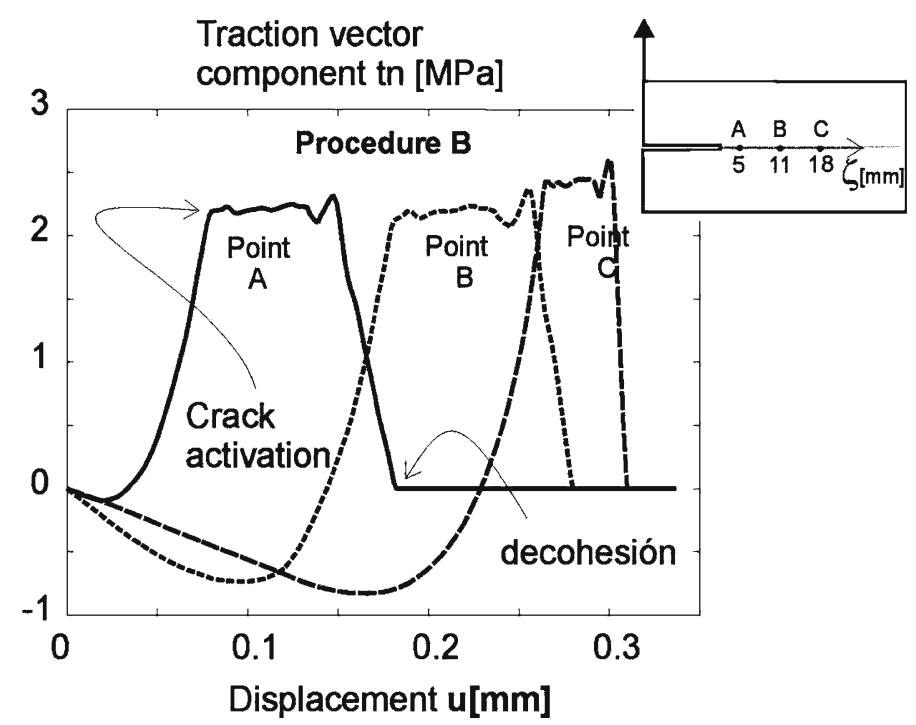

Figure 11. Double cantilever beam test. Stress stages at different material points

is allowed) show, on the contrary, consistent stress profiles. They are not spike-shaped, as in the previous case, and the maximum stress level remains always below $\sigma_{u}$, even for the relatively coarse mesh used in the analysis.

\subsubsection{Fracture stages}

Figure 11 shows the evolution of the traction normal component, $t_{n}$, provided by the CSDA using the previous Procedure B, at three different points A, B and C in the cohesive zone. The abscissa of these curves is the vertical displacement of the load application point, which defines the loading process evolution. There, typical stages in the fracturing process of a material point can be clearly observed: (i) the elastic pre-critic stage, before crack activation, showing increasing values of the stress; (ii) a plateau in the stress evolution corresponding to the stable damage stage in the fracture process zone; (iii) the cohesive stage characterized by the descending branch and (iv) the stress released stage characterized by the final horizontal branch.

\section{Conclusions}

Along this work, the ability of the CSDA to capture fracture phenomena has been examined. The additional contribution of the CSDA to simulating fracturing processes lies on its intrinsic capability to describe in the same modeling setting (i.e. a unique continuum constitutive model), the dissipation phenomena at both the bulk material and the crack interface. This is achieved by introducing the following specific ingredients:

- The strong discontinuity kinematics (7), the inner traction continuity Equation (8) and the continuum softening regularization (9).

- A more precise description of the fracture process zone in terms of the stable damage zone and the cohesive zone (see Figure 4). Unlike in the standard cohesive models, the continuum format of the CSDA allows considering dissipation 


\section{A.E. Huespe et al.}

in softening materials in a stable (non-localized) mode before singularity of the localization tensor, signaling the onset of a strain discontinuity, takes place (Oliver and Huespe, 2004). One can take advantage of this fact to model that part of the fracture process zone were bulk dissipation occurs which, as it was remarked in Bazant (1996), is not done in standard cohesive models. The specific sizes and influence of both zones can be, then, modeled, for every material, in terms of the following properties defining the softening rule in the continuum model:

- The classical fracture energy, $G_{f}$, that governs the total size of the fracture process zone.

- The initial continuum softening modulus, $H_{\text {con }}$, an additional fracturing property to be experimentally determined, that governs the size of the stable damage zone and the corresponding dissipation phenomena at the material bulk. The greater is $\left|H_{\text {con }}\right|$ the smaller is the stable damage zone, $\ell_{d}$ and, therefore, the total dissipated energy is more approximately described by $G_{f}$.

- The use of specific finite elements with embedded discontinuities. They allow capturing the crack propagation, inside the elements, along a crack path that is not $a$ priori determined.

The degeneration of the selected continuum constitutive model into a projected traction-separation law at the fracture interface provides a direct link of the CSDA with the classical cohesive models. In this context, it has been shown that some drawbacks and inconsistencies of simulations using standard cohesive models and reported in the literature can be overcome, i.e.:

- The lack of time continuity of the traction-separation law and its numerical oscillatory character.

- The stress mismatch effect and the lack of smoothness of the stress field near the crack tip.

- The inconsistency of the numerical obtained stress profiles exceeding the peak stress.

\section{References}

Alfano, G. and Crisfield, M.A. (2001). Finite element interface models for the delamination analysis of laminated composites: Mechanical and computational issues. International Journal for Numerical Methods in Engineering 50, 1701-1736.

Bažant, Z.P. (1983). Crack band theory for fracture of concrete presented at Materiaux et Constructions.

Bažant, Z.P. (1996). Analysis of work-of-fracture method for measuring fracture energy of concrete. Journal of Engineering Mechanics-Asce 122, 138-144.

Bažant, Z.P. (2002). Concrete fracture models: testing and practice. Engineering Fracture Mechanics 69, $165-205$.

Bažant, Z.P. and Li, Y.N. (1997). Cohesive crack with rate-dependent opening and viscoelasticity: I. mathematical model and scaling. International Journal of Fracture 86, 247-265.

Bažant, Z.P. and Planas, J. (1988). Fracture and size effect in concrete and other quasibrittle materials: CRC Press.

Elices, M., Guinea, G.V., Gomez, J. and Planas, J. (2002). The cohesive zone model: advantages, limitations and challenges. Engineering Fracture Mechanics 69, 137-163.

Hillerborg, A. (1985). Numerical methods to simulate softening and fracture of concrete. in Fracture Mechanics of Concrete: Structural Application and Numerical Calculation, G. C. S. a. A. D. Tomaso, Ed., pp. 141-170. 
Mosler, J. and Meschke, G. (2003). 3D modelling of strong discontinuities in elastoplastic solids: fixed and rotating localization formulations. International Journal for Numerical Methods in Engineering 57, 1553-1576.

Oliver, J. (1996a). Modelling strong discontinuities in solid mechanics via strain softening constitutive equations 1. Fundamentals. International Journal for Numerical Methods in Engineering 39, 3575 3600.

Oliver, J. (1996b). Modelling strong discontinuities in solid mechanics via strain softening constitutive equations 2. Numerical simulation. International Journal for Numerical Methods in Engineering 39, $3601-3623$.

Oliver, J. (2000). On the discrete constitutive models induced by strong discontinuity kinematics and continuum constitutive equations. International Journal of Solids and Structures 37, 7207-7229.

Oliver, J., Cervera, M. and Manzoli, O. (1999). Strong discontinuities and continuum plasticity models: the strong discontinuity approach. International Journal of Plasticity 15, 319-351.

Oliver, J., Huespe, A.E. (2004). Continuum approach to material failure in strong discontinuity settings. Computer Methods in Applied Mechanics and Engineering 193, 3195-3220.

Oliver, J. and Huespe, A.E. (2004). Theoretical and computational issues in modelling material failure in strong discontinuity scenarios. Computer Methods in Applied Mechanics and Engineering 193, 29873014.

Oliver, J., Huespe, A.E., Pulido, M.D.G. and Chaves, E. (2002). From continuum mechanics to fracture mechanics: the strong discontinuity approach. Engineering Fracture Mechanics 69, 113-136.

Oliver, J., Huespe, A.E., Pulido, M.D.G. and Samaniego, E. (2003). On the strong discontinuity approach in finite deformation settings. International Journal for Numerical Methods in Engineering 56, 10511082.

Oliver, J., Huespe, A.E., Samaniego, E. and Chaves, E.W.V. (2004). Continuum approach to the numerical simulation of material failure in concrete. International Journal for Numerical and Analytical Methods in Geomechanics 28, 609-632.

Ortiz, M. and Pandolfi, A. (1999). Finite-deformation irreversible cohesive elements for three-dimensional crack-propagation analysis. International Journal for Numerical Methods in Engineering 44, 1267 1282.

Papoulia, K.D., Sam, C.H. and Vavasis, S.A. (2003). Time continuity in cohesive finite element modeling. International Journal for Numerical Methods in Engineering 58, 679-701.

Planas, J., Cendon, D., Sancho, J.M., Reyes, E. and Gálvez, J. (2004). Análisis de la respuesta de elementos finitos de intercara en la simulación numérica de fisuras cohesivas. presented at Métodos Computacionais em Engenharia, Lisboa, May 31-June 2, Portugal.

Planas, J., Elices, M., Guinea, G.V., Gomez, F.J., Cendon, D.A. and Arbilla, I. (2003). Generalizations and specializations of cohesive crack models. Engineering Fracture Mechanics 70, 1759-1776.

Rots, J.G. (1988). Computational Modeling of Concrete Fractures: Delft University of Technology.

Simone, A. (2004). Partition of unity-based discontinuous elements for interface phenomena: computational issues. Communications in Numerical Methods in Engineering 20, 465-478.

Simo, J., Oliver, J. and Armero, F. (1993). An analysis of strong discontinuities induced by strainsoftening in rate-independent inelastic solids. Computational Mechanics 12, 277-296.

Willam, K. and Sobh, N. (1987). Bifurcation analysis of tangential material operators. in Transient/Dynamic Analysis and Constitutive Laws for Engineering Materials. vol. 2, G.N. Pande and J. Middleton, Eds.: Martinus-Nijhoff Publishers. pp. C4/1-13.

Zi, G. and Belytschko, T. (2003). New crack-tip elements for XFEM and applications to cohesive cracks. International Journal for Numerical Methods in Engineering 57, 2221-2240. 